\title{
Half Empty Versus Half Full: Linguistic Effects on Numerical Perceptions
}

\author{
Christopher Lee
}

\begin{abstract}
Marketing is about more than just a number. From a long road trip to a half empty arena, adjectives carry numerical associations. The research within this paper builds on that idea while focusing on markedness, a linguistics theory, which has been called the evaluative superstructure of language. Asking "How tall is the person?" is not an indication that the person is tall but merely a neutral way to ask about a person's height. Asking "How short is the person?" however implies the person is actually short in addition to asking for their height. This paper shows how a marked term, such as half empty, results in significantly different, and lower, estimates of attendance than an unmarked term, such as halffull, in addition to influencing other aspects of the consumer judgment scenario. The research contributes to theories on linguistics and consumer judgment by showing how mathematically equivalent frames result in different numerical perceptions.
\end{abstract}

\section{References available upon request.}

C. Lee $(\bowtie)$

Temple University, Philadelphia, PA, USA

e-mail: chrislee@temple.edu 